\title{
First Demonstration of OFDM ECDMA for Low Cost Optical Access Networks
}

\author{
X. Guo ${ }^{1}$, Q. Wang ${ }^{1,3}$, X. Li ${ }^{1}$, L. Zhou ${ }^{2}$, L. Fang ${ }^{2}$, A. Wonfor ${ }^{1}$, J. L. Wei ${ }^{1}$, J von Lindeiner ${ }^{1}$, R. V. Penty ${ }^{1}$, I. H. White \\ ${ }^{1}$ Centre for Photonic Systems, Electrical Engineering Division, Department of Engineering, University of Cambridge \\ 9 J. J. Thomson Avenue, Cambridge, CB3 OFA, UK \\ ${ }^{2}$ Huawei Technologies, Bantian, Longgang District, Shenzhen 518129, China \\ ${ }^{3}$ Department of Electronic Engineering, Tsinghua University, Beijing 100084, China \\ *Corresponding author:xg218@cam.ac.uk \\ Received Month X, XXXX; revised Month X, XXXX; accepted Month X, \\ XXXX; posted Month X, XXXX (Doc. ID XXXXX); published Month X, XXXX
}

\begin{abstract}
We demonstrate for the first time an analogue orthogonal frequency division multiplexing (OFDM) based electrical code division multiplexing access (ECDMA) passive optical network (PON) for the next-generation access applications. Advantages of the system include low cost, high capacity and enhanced spectral efficiency. A proof-of-principal 16 QAM OFDM ECDMA PON downlink experiment is used to show the transmission of an aggregate data rate of $24.8 \mathrm{~Gb} / \mathrm{s}$ within a 8-user system. Transmission is achieved over $25 \mathrm{~km}$ of single-mode telecommunications fibre (SMF) with negligible dispersion and crosstalk penalties.

(C) 2013 Optical Society of America

OCIS Codes: (060.4250) Networks; (060.4510) Optical communications; (060.4080) Modulation

http://dx.doi.org/10.1364/OL.99.099999
\end{abstract}

The expansion of high-speed broadband networks and continuing growth of internet traffic due to high bandwidth internet applications such as 3DTV, online gaming, video telephony and cloud computing have been placing huge bandwidth demands on telecommunication networks [1]. Optical broadband access networks have emerged to address the challenges by sharing channel capacity between customers and then assigning adequate capacity according to service requirements. These are projected to support high data rate, broadband multiple services, scalable bandwidth and flexible communications for multiple end-users. Using this approach, Next Generations Passive Optical Networks (NG-PON) are forecast to increase the global capacity of broadband access well beyond $40 \mathrm{~Gb} / \mathrm{s}$ downlink and $10 \mathrm{~Gb} / \mathrm{s}$ uplink levels. CDMA techniques for optical core and access networks have been widely investigated over the last two decades. However the tradeoff between the high speed and low cost in PONs becomes a major challenge when the total data rate per optical wavelength reaches $40 \mathrm{~Gb} / \mathrm{s}$. Here most all-optical CDMA (OCDMA) solutions proposed are likely to be costly as they use dedicated optical encoders and decoders, complex sources and highpower nonlinear optics for optical thresholding [2]. Hence ECDMA which realizes CDMA coding and decoding in the analogue domain has been proposed [3] which can decrease the bandwidth requirement of the analogue-todigital converter or digital-to-analogue converter (ADC/DAC) greatly. This also can potentially be a low cost, low power consumption and low complexity solution. For example, in ECDMA it is also possible to use a single standard source and detector to support all users as they share a single wavelength. In addition, the system coding and decoding processes have the potential to improve receiver sensitivity and increase power budgets due to coding gain, which potentially allows optimization of existing infrastructure, simplifies network management and further reduces the cost of an upgraded PON system. Recently, ECDMA with chip rates up to 18 Gchip/s were implemented using electronic finite impulse response (FIR) transversal filters (TF) with $1.25 \mathrm{~Gb} / \mathrm{s}$ Non-return-to zero (NRZ) modulation [3]. However, as the data rates increase, the computational requirements involved in electronic dispersion compensation for such serial modulation format become very complicated. To combat optical modal dispersion, OFDM has been proposed as an effective technique to deal with linear signal distortions and widely adopted in wireless and broadcast systems that have dispersive fading channels [4]. It is also a promising alternative to Time Division Multiplexing (TDM) and Wavelength Division Multiplexing (WDM) in that it avoids the need for burst mode operation and also uses only a single wavelength. It also has the potentials to significantly reduce the required bandwidth of electronic stages at the user location such as Optical Network Unit (ONU), thus reducing its complexity and cost. Of course, OFDM in optical communications have recently gained worldwide research [5]. For example, Zhang et al have experimentally demonstrated record high $19.25 \mathrm{~Gb} / \mathrm{s}$ realtime end-to-end OFDM transmissions over 25km SMF [6]. Chen et al have demonstrated a $40 \mathrm{~Gb} / \mathrm{s}$ optical OFDM transmission of $20 \mathrm{~km}$ SMF using a $10 \mathrm{GHz}$ bandwidth EAM [7]. OFDM involves in parallel modulation in which Fast Fourier Transform (FFT)-based implementation enables an adaptation of the modulation formats on each OFDM subcarrier that the system can adapt to imperfect 
system conditions with hybrid bandwidth allocation. OFDM also can provide high signal transmission capacity and high spectral efficiency and excellent resistance to chromatic dispersion and polarization mode dispersion. If the OFDM based transmitter and receiver is encoded and decoded with orthogonal codes, it can take advantage of both the dense subcarrier spacing of OFDM and the multiple access benefits of CDMA. Easy equalization of OFDM and crosstalk cancellation techniques in CDMA are also of benefit. Hence based on the separate advantages of ECDMA and OFDM, the combination of the CDMA and OFDM could be an important evolution route for future low cost and high speed PONs. Although digital implementations are feasible (i.e. as in [8]), but low cost solutions demand analogue implementations. This is therefore the first demonstration of an analogue OFDM ECDMA system that features low cost electrical encoders and decoders that greatly decrease the overall cost and complexity. The gross data rate $3.1 \mathrm{~Gb} / \mathrm{s}$ per user OFDM ECDMA signals for two ONUs are transmitted in this PON system to validate the architecture feasibility. Error free transmission is demonstrated in the experiment over a $25 \mathrm{~km}$ SMF downlink.

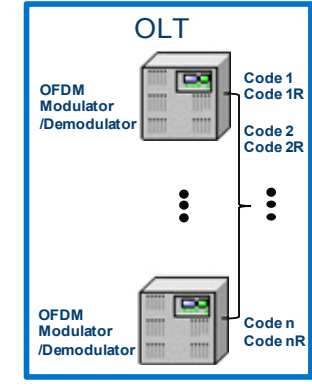

Multiple electronic CDMA

encoder and decoder

Fig. 1 Architecture of a OFDM-ECDMA PON

The proposed OFDM-ECDMA PON architecture is shown in Fig. 1. For downlink operation, the optical line terminal (OLT) generates unique encoded OFDM data streams for each user and these are simultaneously transmitted. At the receiver, each residential user has a corresponding unique ECDMA modem which decodes its own data while rejecting the data from other users. The decoded data is then demodulated by OFDM modem. A similar process can be employed in the uplink. This architecture provides several benefits including improved spectral efficiency, simple implementation, bidirectional transmission, coding gain and networking functions such as service monitoring, multicasting and switching. In particular, it is possible to use a single standard source and detector at the central office to support all users as they share a single wavelength.

The electrical CDMA implementation of the encoder and decoder are realized by an electronic FIR TF provided by Avago Technologies, and the schematic is shown in Fig.
2 (a). The FIR TF splits an input pulse across taps each of which is separated by a time delay. In this work, the taps of the FIR TF are used to generate bipolar sequences (consisting of chips with weights of +1 and -1) and tap weights can also be electronically modified to fractional values. 9 tap FIR TFs are used in this experiment. In order to characterize the FIR filter, a 25-ps electrical pulse is input to the device and the tap weights are set to be [+1 $+1+1+1-1-1-1-1]$. The resulting time domain response is shown in Fig. 2(b). The measured tap spacing between chips is $78 \mathrm{ps}$ and corresponds to a chip rate of 12.8 Gchips/s. This technology makes possible low cost and low power CDMA generation at high chip rates.
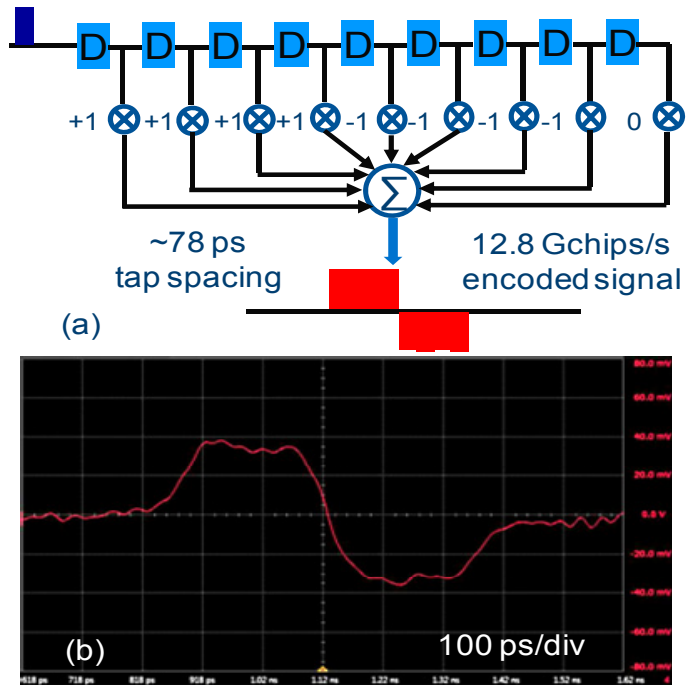

Fig. 2. (a) Schematic of the FIR TF for electrical CDMA, where D Is the associated delay element (b) 12.8 Gchips/s FIR TF Time domain response for code generation of $[+1+1+1+1-1-1-1-1]$.

\section{Table 1 OFDM ECDMA Experimental Parameters}

\begin{tabular}{ll} 
OFDM parameters & \\
\hline Number of bits & $1.24 \times 10^{5}$ \\
FFT size & 64 \\
Subcarrier bandwidth & $25 \mathrm{MHz}$ \\
Symbol rate & $1.6 \mathrm{Gs} / \mathrm{s}$ \\
Modulation/ Subcarriers & $16 \mathrm{QAM} / 2-32$ \\
Training sequences & $200 \times 64$ \\
Self correlation synchronization size & 64 \\
CP ratio & 0.2 \\
\hline \hline
\end{tabular}

Table 1 shows the OFDM configuration for the experiments. The data is mapped to 16-Quadrature Amplitude Modulation (16-QAM) symbols and then input to the IFFT with a 64 -subcarrier vector. The subcarriers are deliberately arranged to be the 2 subcarriers close to the optical carrier dropped off and the other 62 subcarriers formed Hermitian symmetry with the first 31 subcarriers in the positive frequency bins that are used to carry user data, so that IFFT output is real-valued, although will lose half spectral efficiency but can realize intensity modulation-direct detection (IM-DD) in optical 
system and simplify the configuration of the experiment. In this experiment, each user gross data rate of $3.1 \mathrm{~Gb} / \mathrm{s}$ is mapped into 16 QAM modem with symbol rate of $1.6 \mathrm{Gs} / \mathrm{s}$ limited only by the FIR TF response. After implementing the IFFT, a cyclic prefix (CP) of $20 \%$ is adopted in front of each OFDM symbol to mitigate the inter-symbol interference (ISI). The 200×64 training sequences (cover all the data subcarriers) have been added in front the whole OFDM sequences for the channel coefficients estimation of individual subcarriers and make possible simple one-tap equalization. 64 bits preamble symbols that utilizing the strong cross-correlation properties of Pseudo-noise $(\mathrm{PN})$ sequences are also added in front the whole OFDM symbol sequences for system automatic timing synchronization. The OFDM data is generated offline by Matlab and output by Tektronix Arbitrary Waveform Generator (AWG) 70002A with maximum sampling rate of $25 \mathrm{GSamples} / \mathrm{s}$.

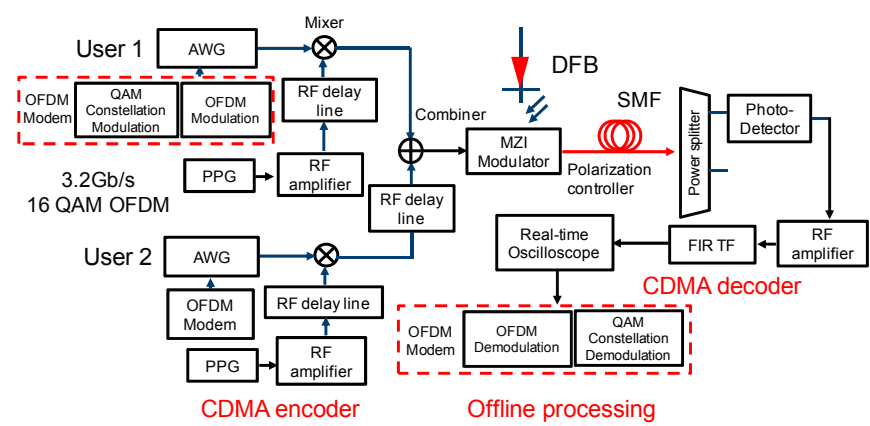

Fig. 3 Experimental setup for two $3.1 \mathrm{~Gb} / \mathrm{s}$ OFDM ECDMA users with $12.8 \mathrm{Gchips} / \mathrm{s}$ encoders and decoders.

The experimental setup is shown in Fig. 3. To provide a proof-of-principle demonstration, two users out of an 8user system and for the downlink direction are measured. Each user has a different 8 bit Walsh-Hadamard code. In this demonstration, two codes are chosen with tap configurations [+1 +1 +1 +1 - $1-1-1-1]$ (code 5) and [+1 +1 $-1-1-1-1+1+1$ ] (code 7) respectively. The OFDM symbol rate of $1.6 \mathrm{Gs} / \mathrm{s}$ per user with $16 \mathrm{QAM}$ modulation is generated by an AWG before being mixed with codes produced for reasons of experimental convenience by a Pulse Pattern Generator (PPG). The resulting two electrical CDMA signals are combined together using a 26 $\mathrm{GHz}$ bandwidth radio-frequency (RF) mixer and then are used to optically modulate a $1550 \mathrm{~nm}$ continuous-wave (CW) signal from a distributed-feedback laser (DFB) via a $\mathrm{LiNbO}_{3} \mathrm{Mach}^{-Z e h n d e r}$ modulator (MZM). The DFB laser is an ILX Lightwave 79800D C-band module with full power of $10 \mathrm{dBm}$ and wavelength/attenuation accuracy of $\pm 0.05 \mathrm{~nm} / \pm 0.1 \mathrm{~dB}$. The modulated optical signal is transmitted through $25 \mathrm{~km}$ of standard SMF and detected by a receiver with $-15 \mathrm{dBm}$ sensitivity at $10 \mathrm{~Gb} / \mathrm{s}$ (NRZ). The resulting signal is amplified by an RF amplifier. Each user's data is decoded from the ECDMA signal using correlation performed by a separate 12.8 Gchips/s and 78 ps tap spacing FIR TF. For decoding, due to the orthogonal nature of the Walsh-Hadamard codes, it is necessary to use the time inverse of the transmitted code $5 \mathrm{R}[-1-1-1-1+1+1+1+1]$ in the decoder so that when the code is appropriate, the desired received decoded signal will form autocorrelation peaks at the optimal sampling point and the interference of the other ONU can be eliminated. The output is captured using a real-time oscilloscope (RTO). In the experiments, a $4 \mathrm{GHz}$ bandwidth RTO from Keysight is used in order to minimize the noise.

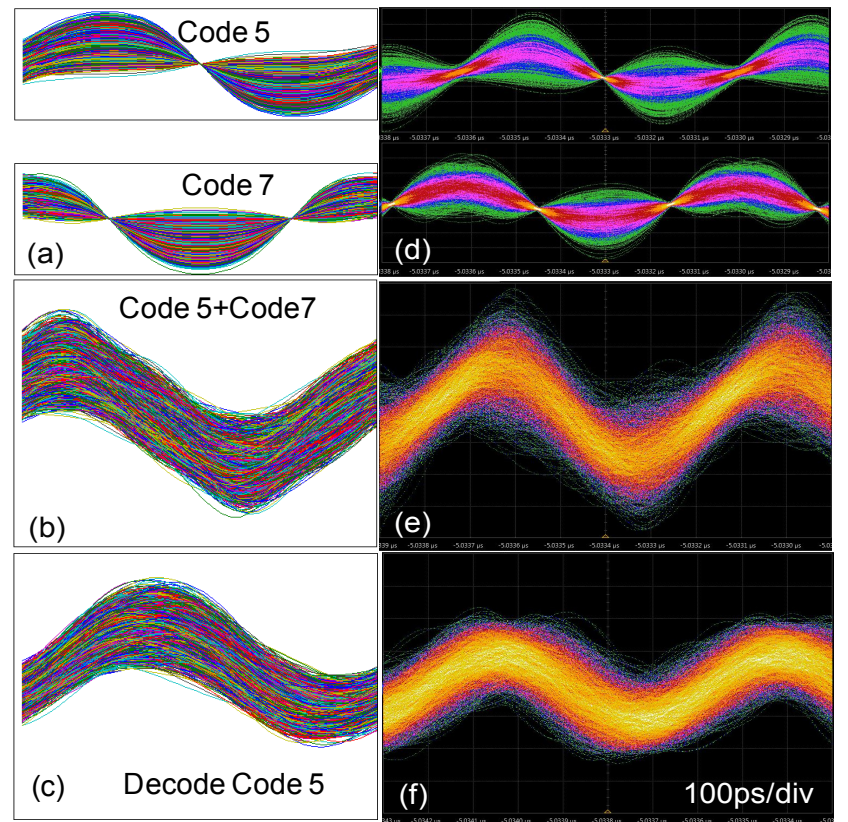

Fig. 4 (a-c) Simulated simulation $3.1 \mathrm{~Gb} / \mathrm{s} 16$ QAM OFDM symbols with two users encoded with code 5 and code 7 , combined signal (sum of the encoded signals using code 5 and code 7 ) and decoded signal using code $5 \mathrm{R}$ with interference from the other user. (d-f) corresponding experimental results with encoding, mixing and decoding scenarios.

To visualize the encoding and decoding process of OFDM symbols, simulations are carried out with the same channel data rate and CDMA parameters as used in the experiments, with the encoding, mixing and decoding processes shown in Fig.4 (a-c). The simulation results conform well to the experimental results. Fig. 4(d) shows a plot of the synchronized superposition of the encoded 3.1 Gb/s 16 QAM OFDM symbols using code 5 and code 7 respectively. Fig.4 (e) shows the aligned and combined two encoded signals. The signal decoded for code 5 for the link is illustrated in Fig.2 (f). In Fig. 2 (b-c) and (e-f) the mixing and decoding waveforms, the modulating wave shape is due to the autocorrelation process of the DC components from modulating the MZM. The received signal is captured in RTO and offline processed in Matlab for time synchronization and OFDM demodulation. The received OFDM symbols are synchronized by the self correlation of PN synchronization sequences and then equalized by the one-tap equalizer from the channel 
response. The channel response for different subcarriers are measured to be relatively flat and the maximum power difference is within $2 \mathrm{~dB}$, as shown in Fig. 5 (a), hence in order to simplify the experiment, all the subcarriers are modulated at $16 \mathrm{QAM}$ and the adaptive power/bit loading is not applied. Fig. 5 (b) shows the 16 QAM constellation demodulated 16 QAM signals of the two users within the 8-user system at a received optical power of $-5 \mathrm{dBm}$, the bit error rate (BER) is measured to be $2.4 \times 10^{-4}$.
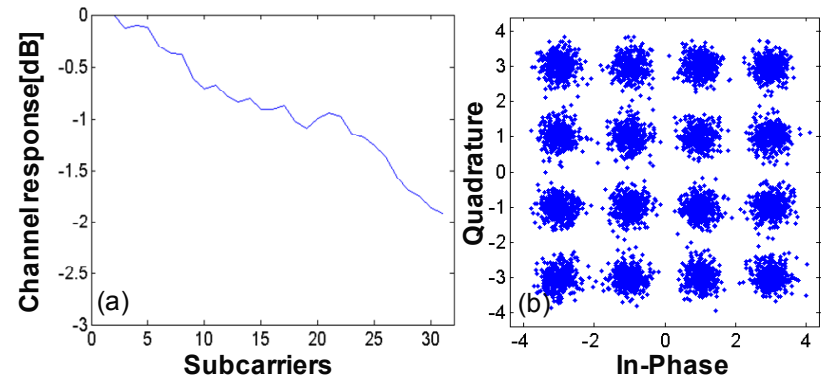

Fig. 5 (a) Channel response for different subcarrers and (b) demodulated 16 QAM OFDM symbols after $25 \mathrm{~km}$ SMF at received optical power of $-5 \mathrm{dBm}$ with BER measured $2.4 \times 10^{-4}$

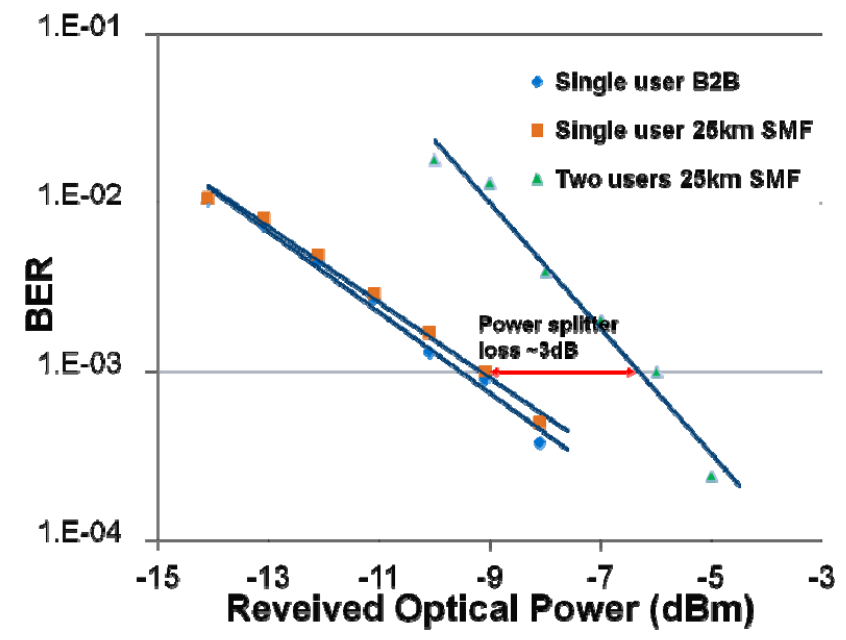

Fig. 6 BER performance of single CDMA user and two users for back-to-back and after $25 \mathrm{~km}$ SMF transmission

Fig. 6 shows that the BER curves scale with a logarithm to base 10 for single user and two users. The BER curves indicate error free $\left(\mathrm{BER}<10^{-3}\right)$ operation with received optical power of $-9 \mathrm{dBm}$ and $-5.8 \mathrm{dBm}$ for a single user and for two users respectively. Comparing the single user optical back-to-back (B2B) and after $25 \mathrm{~km}$ transmission, there is a negligible $0.1 \mathrm{~dB}$ power penalty (dispersion or equipment penalties) measured, which indicates good dispersion resistance. Given $3 \mathrm{~dB}$ power splitter loss for the two users, there crosstalk penalty for two simultaneous users is measured to be only $0.2 \mathrm{~dB}$ at a BER of $10^{-3}$ due to the link dispersion causing degraded channel orthogonality. The slope difference between one and two users is due to the automatic gain control of FIR TF caused slightly different noise level. The BER of the system could therefore be error free $\left(\mathrm{BER}<10^{-3}\right)$ if implemented with forward error correction (FEC). Errorfree transmission of two users validates the theoretical modelling results, and in turn, this shows the viability of eight ONU channels with a record aggregate date rate of $24.8 \mathrm{~Gb} / \mathrm{s}$ using a $16 \mathrm{QAM}$ OFDM modulation scheme. This can be increased further if advanced orthogonal codes, higher order modulation formats and higher FIR TF chip rates are used. Of course a more complex synchronization scheme will need to be implemented to achieve this performance. This represents a 2.5-fold improvement over the previous demonstration of NRZECDMA [3] without compromising any other performance.

In conclusion, this paper describes a proof-of-principle low-cost high-performance pure analogue OFDM ECDMA PON realized by using electronic transversal filters at $12.8 \mathrm{Gchips} / \mathrm{s}$ using a downlink demonstration experiment. This represents a 2.5 -fold improvement over previous NRZ-ECDMA results [3]. The electrical FIR TF used allows the required $\mathrm{ADC} / \mathrm{DAC}$ to be simpler as the necessity for high cost due to higher bandwidth is removed. The demonstration of error-free transmission of two users within an 8-user system makes such access mechanisms compatible with the current deployed infrastructures where the downlink and uplink signals can be sent in the same wavelength. This shows the advantages of the approach in allowing flexible, nondisruptive use of legacy infrastructure, high data rate transmission, long distance and potential high user numbers. The system is flexible, low cost and energy efficient potentially suitable for second-stage nextgeneration $\mathrm{PON}$ specifications.

We acknowledge D. G. Cunningham and J. D. Ingham (Avago Technologies) for helpful discussions and for providing the transversal filters.

\section{Reference}

1. Y. Luo, X. Zhou, F. Effenberge, X. Yan, G. Peng, Y. Qian, and Y. Ma, J. Lightw. Technol., 31, 587-593 (2013).

2. K. Kitayama, Optical Code Division Multiple Access: A Practical Perspetive (Cambridge University Press, 2014).

3. J. B. Rosas-Fernandez, J. D. Ingham, R. V. Penty, and I. H. White, J. Lightw. Technol., 27, 306-313 (2009).

4. J. Armstrong, J. Lightw. Technol., 27, 189-204 (2009).

5. N. Cvijetic, J. Lightw. Technol., 30, 384-398 (2012).

6. Q. W. Zhang, E. Hugues-Salas, R. P. Giddings, M. Wang, and J. M. Tang, Opt. Express., 21, 9167-9179 (2013).

7. H. Chen, C. Wei, D. Hsu, M. Yuang, J. Chen, Y. Lin, P. Tien, S. Lee, S. Lin, W. Li, C. Hsu, and J. Shih, IEEE Photon. Technol. Lett., 24, 85-87 (2012).

8. L. Zhang, X. Xin, B. Liu, J. Yu, and Q. Zhang, Opt. Express., 18, 18347-18353 (2010). 